\title{
Corela
}

Cognition, représentation, langage

17-2 | 2019

Vol. 17, $\mathrm{n}^{\circ} 2$

\section{De l'interprétation argumentative}

\section{Kohei Kida}

\section{(2) OpenEdition}

\section{Journals}

Electronic version

URL: http://journals.openedition.org/corela/9621

DOI: 10.4000/corela.9621

ISSN: 1638-573X

\section{Publisher}

Cercle linguistique du Centre et de l'Ouest - CerLICO

\section{Electronic reference}

Kohei Kida, "De l'interprétation argumentative", Corela [Online], 17-2 | 2019, Online since 16 December 2019, connection on 21 January 2021. URL: http://journals.openedition.org/corela/9621 ; DOI: https:// doi.org/10.4000/corela.9621

This text was automatically generated on 21 January 2021.

\section{(c) (i) (2) (2)}

Corela - cognition, représentation, langage est mis à disposition selon les termes de la licence Creative Commons Attribution - Pas d'Utilisation Commerciale - Partage dans les Mêmes Conditions 4.0 International. 


\section{De l'interprétation argumentative}

\section{Kohei Kida}

\section{Introduction}

1 La théorie de l'Argumentation dans la Langue, conçue à l'origine par Jean-Claude Anscombre et Oswald Ducrot dans les années 1980 (cf. Anscombre et Ducrot, 1983) pour surmonter les difficultés que soulève toute approche référentielle du sens, repose sur une hypothèse structuraliste selon laquelle les mots renvoient aux mots. La Théorie des Blocs Sémantiques proposée par Marion Carel maintient et même radicalise cette hypothèse en ce qu'elle s'impose de décrire la valeur sémantique d'une entité linguistique par des discours, et seulement par des discours, que cette entité évoque ou modifie, en s'interdisant strictement tout recours à l'extralinguistique. Mais il n'est pas question de considérer tous les types de discours; seuls sont pris en compte les discours argumentatifs. En ce sens, la Théorie des Blocs Sémantiques relève, comme la théorie de l'Argumentation dans la Langue d'ailleurs, de la sémantique argumentative (cf. https://semanticar.hypotheses.org).

2 Le dernier article de Carel (Carel, 2019) marque un tournant dans l'évolution de la théorie dans la mesure où il y introduit trois notions novatrices: celles de «Carte Argumentative du Lexique», de "décodage argumentatif» et d'«interprétation argumentative ». Ces notions permettent en effet de construire des faits nouveaux et de rendre compte de certaines observations, de manière plus raffinée et plus convaincante que dans les versions antérieures de la théorie.

3 Je tenterai ici de développer la notion d'interprétation argumentative qui, telle qu'elle est présentée par Carel, me semble comporter quelques imprécisions. J'essaierai notamment de montrer que la notion peut recouvrir des réalités plus complexes que la définition de Carel ne le laisserait supposer. 


\section{Théorie des Blocs Sémantiques}

4 Je tiendrai pour acquises les hypothèses de base de la théorie, pour lesquelles je me contenterai de renvoyer à des études relativement récentes (Carel, 2011, 2012, 2017 ; Ducrot, 2016; Kida, 2015, 2016, 2017; Nishiwaki, 2016). Je rappellerai néanmoins quelques notions fondamentales que j'utiliserai tout le long de ce travail. J'introduirai d'abord deux objets : enchaînement argumentatif et schéma argumentatif.

6 Par définition, on entendra par «enchaînement argumentatif » le discours argumentatif comportant soit un connecteur du type de donc, soit un connecteur du type de pourtant. Le discours il y avait du danger donc Pierre a pris des précautions en est un exemple. Les discours Jean a pris des précautions parce qu'il y avait du danger et s'il y a du danger Max prendra des précautions sont aussi des enchaînements argumentatifs. Il en est de même pour des discours comme il y avait du danger pourtant Marie n'a pas pris de précaution, Sophie n'a pas pris de précaution bien qu'il y ait du danger, même s'il y a du danger Claire ne prendra pas de précaution, etc.

7 Le schéma argumentatif ${ }^{1}$ est la structure abstraite partagée par une famille d'enchaînements argumentatifs. Il est d'usage de noter un schéma argumentatif en petites capitales. Ainsi, l'enchaînement il y avait du danger donc Pierre a pris des précautions, ainsi que d'autres du même type, sont construits sur le même schéma DANGER DC PRÉCAUTION où DC représente les divers connecteurs du type de donc. Il en est de même pour l'enchaînement il y avait du danger pourtant Pierre n'a pas pris de précaution et bien d'autres du même type qui sont tous construits sur le même schéma DANGER PT NEG PRÉCAUTION où PT représente les divers connecteurs du type de pourtant et NEG est un opérateur de négation argumentative regroupant non seulement la négation syntaxique ne ... pas à l'exclusion de ses emplois métalinguisitques, mais également toutes les expressions à orientation argumentativement négative comme peu, rarement, etc. Lorsqu'un enchaînement est construit sur un schéma, on dira qu'il le " concrétise " (Carel 2017 : 7). Ainsi, l'enchaînement il y a du danger donc Pierre a pris des précautions concrétise le schéma DANGER DC PRÉCAUTION. Nul besoin pour un enchaînement argumentatif de reprendre exactement les mêmes mots du schéma qu'il concrétise. Ainsi, le schéma DANGER DC PRÉCAUTION est concrétisé non seulement par l'enchaînement il y avait du danger donc Pierre a pris des précautions mais également par l'enchaînement $i l$ allait pleuvoir donc Marie est redescendue, la pluie étant vue comme un danger et la redescente, comme une précaution.

Par ailleurs, la Théorie des Blocs Sémantiques inclut un ensemble cohérent d'hypothèses. Je me limiterai à en présenter deux, parmi les plus essentielles.

9 Première hypothèse: tout énoncé est paraphrasable par des enchaînements argumentatifs attachés aux schémas qu'ils concrétisent. Lorsqu'un énoncé est paraphrasable par un enchaînement, on dira que l'énoncé « évoque » l'enchaînement (Carel 2017 : 6). Ainsi, l'énoncé Pierre a été prudent évoque l'enchaînement il y avait du danger donc Pierre a pris des précautions. Le même énoncé évoque par ailleurs dans certaines circonstances l'enchaînement Pierre a été prudent donc Marie a été en sécurité et dans d'autres, l'enchaînement Pierre a été prudent pourtant Marie n'a pas été en sécurité. Par ailleurs, notons que, pour décrire sémantiquement un énoncé par un enchaînement, il est indispensable d'attacher ce dernier à un schéma qu'il concrétise. Soit l'énoncé la vue de cette maison me fit tressaillir, inspiré de la nouvelle de Maupassant 
À vendre. Il ne suffit pas, pour décrire sémantiquement l'énoncé, de constater qu'il évoque l'enchaînement je vis cette maison donc je tressaillis. Il faut en plus attacher cet enchaînement à un schéma qu'il concrétise. Ainsi, une chose est de l'attacher au schéma JOIE DC TRESSAILLIR, autre chose est de l'attacher au schéma PEUR DC TRESSAILLIR.

Deuxième hypothèse : les mots pleins (nom, adjectif, verbe, etc.) ont souvent pour fonction de fournir un schéma sur lequel sont construits des enchânements. Le mot prudent peut fournir des schémas comme DANGER DC PRÉCAUTION, PRUDENT DC SÉCURITÉ, PRUDENT PT NEG SÉcURITÉ. Lorsqu'un mot fournit un schéma, on dira que le mot " préfigure » le schéma s'il présente une alternative entre le schéma et un autre ; sinon, on dira que le mot «signifie» le schéma (Carel 2019: 4). Le mot prudent signifie le schéma DANGER DC PRÉCAUTION, et préfigure les schémas PRUDENT DC SÉCURITÉ Ou PRUDENT PT NEG SÉCURITÉ. Par ailleurs, lorsqu'un enchaînement est construit sur un schéma, on appellera "constitutif» le terme qui fournit le schéma. Ainsi, le mot prudent dans l'énoncé Pierre a été prudent est le terme constitutif lorsqu'il fournit le schéma DANGER DC PRÉCAUTION que concrétise l'enchaînement il y avait du danger donc Pierre a pris des précautions évoqué par l'énoncé.

\section{Nouvelles notions}

11 Carel (2019) présente sa conception originale de la Carte Argumentative du Lexique, du décodage argumentatif et de l'interprétation argumentative. Je vais la résumer rapidement, illustrée sur des exemples partiellement choisis par mes soins, afin de montrer que l'interprétation argumentative soulève quelques interrogations dont il sera question par la suite.

\subsection{Carte Argumentative du Lexique}

La Carte Argumentative du Lexique est une représentation graphique dans laquelle les

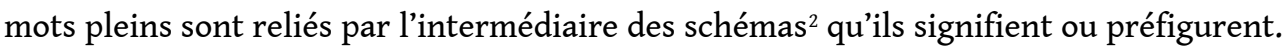
Les mots y sont représentés comme des villes et les schémas, comme des routes qui mènent d'une ville à l'autre.

13 En voici quelques exemples. Soit le mot prudent. Il signifie entre autres le schéma DANGER DC PRÉCAUTION et il est relié, par l'intermédiaire de ce schéma, au mot danger. Le mot prudent préfigure par ailleurs entre autres le schéma PRUDENT DC SÉCURITÉ et il est relié, par l'intermédiaire de ce schéma, au mot sécurité. Soit encore le mot guerre. Il est relié notamment aux mots menacé ou précieux respectivement par l'intermédiaire des schémas MENACÉ DC SE BATTRE ou PRÉCIEUX PT DÉTRUIRE qu'il signifie.

La compréhension des énoncés consiste à parcourir la Carte Argumentative du Lexique et ce, dans deux directions opposées (Carel 2019:9) : en allant des mots aux schémas (il y a alors décodage argumentatif) ou des schémas aux mots (il y a alors interprétation argumentative).

\subsection{Décodage argumentatif}

Le décodage argumentatif d'un énoncé consiste à déterminer les enchaînements qu'il évoque et le terme constitutif qu'il comporte, à parcourir la Carte Argumentative du 
Lexique pour déterminer les schémas que le terme constitutif signifie ou préfigure, et enfin à attacher les enchaînements aux schémas.

Soit l'énoncé le petit enfant voyait l'inconnu approcher, exemple inspiré de la nouvelle de Maupassant Le Retour. Il évoque entre autres l'enchaînement le petit enfant regardait l'inconnu et donc savait qu'il approchait. Le verbe voir dans l'énoncé est le terme constitutif, qui signifie le schéma REGARDER DC SAVOIR, auquel est attaché l'enchaînement. Considérons encore le passage Jean Valjean avait la prudence de ne jamais sortir le jour (Hugo, Les Misérables, tome II, livre quatrième, chapitre IV). Jean Valjean loue à Paris une maison, la masure Gorbeau, où il se cache avec Cosette. Le passage évoque notamment l'enchaînement il pouvait être vu le jour donc il ne sortait jamais le jour. Le mot prudence est le terme constitutif, qui signifie le schéma DANGER DC PRÉCAUTION, concrétisé par l'enchaînement, la possibilité d'être vu étant décrite comme un danger et la décision de ne jamais sortir le jour, comme une précaution.

\subsection{Interprétation argumentative}

L'interprétation argumentative d'un énoncé consiste à déterminer les enchainements argumentatifs qu'il évoque et à parcourir la Carte Argumentative du Lexique pour déterminer les schémas concrétisés par ces enchaînement et les mots qui signifient ou préfigurent ces schémas, ces mots étant choisis comme des termes constitutifs implicites. Elle peut éventuellement amener à parcourir encore la Carte Argumentative du Lexique pour déterminer d'autres schémas signifiés ou préfigurés par les termes constitutifs implicites et les enchaînements qui concrétisent ces nouveaux schémas.

Soit un exemple extrait de la nouvelle de Maupassant Boule de Suif. Les voyageurs font un arrêt dans une auberge de Tôtes, occupée par les Prussiens. Le comte Bréville, CarréLamadon et Loiseau sont reçus par l'officier qui, étendu dans un fauteuil, reste silencieux :

Au bout de quelques instants il dit enfin:

- «Qu'est-ce que fous foulez?»

Le comte prit la parole : - Nous désirons partir, monsieur.

19 Une lecture possible du segment Le comte prit la parole consiste à dire qu'il évoque l'enchaînement il était devant l'officier pourtant il prit la parole. À quel schéma peut-on l'attacher alors? Deux possibilités au moins se présentent. On peut d'abord supposer que l'enchaînement concrétise le schéma NEG ENVIE PT FAIT. Ce schéma peut être signifié par le terme constitutif implicite courageux et le narrateur pourrait dire du comte il fut courageux. On peut aussi supposer que l'enchaînement concrétise le schéma RISQUÉ PT FAIT. Ce schéma peut être signifié par le terme constitutif implicite audacieux et le locuteur serait prêt à dire du comte il fut audacieux. On peut même aller plus loin. Dans le cas du schéma NEG ENVIE PT FAIT, signifié implicitement par le mot courageux, celui-ci préfigure par ailleurs le schéma COURAGEUX DC ADMIRÉ et le narrateur aurait pu dire du comte il fut courageux donc il fut admiré par ses compagnons.

\subsection{Bilan}

On aura compris que le décodage argumentatif et l'interprétation argumentative ont cela de commun que l'un et l'autre amènent à déterminer, pour comprendre un énoncé, le terme constitutif et en conséquence le schéma qu'il signifie ou préfigure. Ce qui les 
distingue, c'est que le terme constitutif est explicitement formulé dans le cas du décodage argumentatif alors qu'il est implicite dans le cas de l'interprétation argumentative ${ }^{3}$. D'où la remarque de Carel $(2019: 8)$, selon laquelle « le lecteur reste en partie libre » quant au choix de son interprétation argumentative. Le lecteur de Boule de Suif est libre d'attacher l'enchaînement il était devant l'officier pourtant il prit la parole évoqué par l'énoncé Le comte prit la parole soit au schéma NEG ENVIE PT FAIT, soit au schéma RISQUÉ PT FAIT, soit encore à n'importe quel autre, quitte à revenir ultérieurement sur le schéma choisi pour le modifier de telle manière qu'il soit mieux adapté à la compréhension globale du texte.

\section{Variétés d'interprétation}

21 Carel insiste à juste titre sur la liberté du lecteur quant à ses interprétations textuelles. L'observation des faits montre cependant que l'interprétation argumentative peut prendre diverses formes selon que le locuteur a l'intention plus ou moins manifeste d'attacher l'enchaînement évoqué par son énoncé à un schéma précis, sans toutefois formuler explicitement de terme constitutif qui puisse fournir le schéma. Je me propose d'examiner un certain nombre de cas.

\subsection{Absence de schéma}

Je commencerai par étudier l'exemple suivant, le titre d'un article du journal Le Monde daté du 10 mai $2019^{4}$ :

(1) Football: Neymar suspendu trois matchs après son altercation avec un supporter

Je rappelle la situation. Après la finale de la aCoupe de France perdue par le Paris-SaintGermain contre le Stade rennais le 27 avril 2019, Neymar, attaquant brésilien du club parisien, monte les marches du Stade de France chercher sa médaille. Il est pris à partie avec des insultes par un spectateur, à qui il assène un coup au visage. La Commission de discipline de la Fédération Française de Football ouvre une procédure à son encontre. Elle annonce le 10 mai sa décision : trois matchs de suspension ferme et deux matchs avec sursis.

Dans ces conditions, l'énoncé Neymar suspendu trois matchs après son altercation avec un supporter en (1) peut recevoir trois interprétations au moins.

La première interprétation consiste à dire que l'énoncé évoque l'enchaînement Neymar a eu une altercation avec un supporter donc il est suspendu trois matchs et cet enchaînement concrétise le schéma FAUTE DC SANCTION. L'altercation de Neymar est ainsi vue comme une faute et sa suspension, comme une sanction. Cette interprétation amène par ailleurs à supposer que le schéma est signifié implicitement par le mot juste, ce qui permettrait de dire par exemple la Commission a été juste.

Selon la deuxième interprétation, l'énoncé Neymar suspendu trois matchs après son altercation avec un supporter évoque l'enchaînement Neymar a eu une altercation avec un supporter pourtant il est suspendu trois matchs, qui concrétise le schéma NEG FAUTE PT SANCTION. Ce qu'il faut bien voir, c'est que l'altercation est ici vue comme une faute légère, l'adjectif léger dans cette expression fonctionnant comme un modificateur déréalisant inverseur (Ducrot, 1995) (on se rappelle que NEG ne recouvre pas seulement 
la négation syntaxique ne ... pas mais aussi toutes les expressions à orientation argumentativement négative). Autrement dit, l'altercation en question n'est pas ici considérée comme une faute grave. Par ailleurs, toujours selon cette interprétation, on suppose que le schéma NEG FAUTE PT SANCTION est signifié implicitement par sévère, et on pourrait dire par exemple la Commission a été sévère.

La troisième interprétation enfin amène à dire que l'énoncé Neymar suspendu trois matchs après son altercation avec un supporter évoque le même enchaînement que le précédent, Neymar a eu une altercation avec un supporter pourtant il est suspendu trois matchs. Ce qui distingue la deuxième et la troisième interprétation, c'est le schéma concrétisé par l'enchaînement. Selon la troisième interprétation, le même enchaînement concrétise non pas le schéma NEG FAUTE PT SANCTION comme dans le cas de la deuxième interprétation, mais le schéma FAUTE PT NEG SANCTION. La suspension de trois matchs est alors vue comme une sanction légère, l'adjectif léger fonctionnant toujours comme inverseur, ce qui reviendrait à dire que Neymar a été peu sanctionné. Cette interprétation, qu'on pourrait accepter plus facilement avec un seulement implicite dans la deuxième partie de l'enchaînement, amène par ailleurs à déterminer le terme constitutif implicite clément et on pourrait dire la Commission a été clémente.

Ces interprétations, ainsi que d'autres, sont théoriquement possibles et on pourrait en conclure comme Carel (2019) que le lecteur est libre de choisir son interprétation. Toutes les interprétations ne sont cependant pas empiriquement possibles. Dans le cas de notre article du journal Le Monde, la lecture de son corps rend vraisemblable de supposer que le locuteur laisse délibérément absent le terme constitutif ${ }^{5}$, que ce soit ou non par souci de neutralité journalistique, si neutralité il y a. Le texte est en effet composé d'un ensemble d'énoncés qui, sinon objectifs en eux-mêmes, prétendent l'être $\mathrm{du}$ moins. D'où une première constatation: l'absence de terme constitutif dans un énoncé amène parfois à déterminer l'enchaînement qu'il évoque sans l'attacher à aucun schéma ${ }^{6}$.

\subsection{Bonne interprétation}

Imaginons que deux fans de football discutent de l'article du journal Le Monde :

(2) X : Incroyable! Neymar suspendu trois matchs après son altercation avec un supporter.

Y : Tu veux dire que la Commission a été sévère?

$\mathrm{X}$ : Oui.

Le locuteur X, vraisemblablement supporter du PSG, reprend dans le titre de l'article la phrase Neymar suspendu trois matchs après son altercation avec un supporter pour produire son propre énoncé. Il a l'intention de produire son deuxième énoncé de telle manière qu'il évoque l'enchaînement Neymar a eu une altercation avec un supporter pourtant il est suspendu trois matchs, qui concrétise le schéma NEG FAUTE PT SANCTION, signifié par le terme constitutif implicite sévère. L'interlocuteur $\mathrm{Y}$ reconnaît cette intention, de sorte qu'il comprend l'énoncé comme évoquant le même enchaînement attaché au même schéma.

31 On peut dire qu'il s'agit ici d'une «bonne » interprétation, dans la mesure où l'interprétation argumentative s'effectue exactement comme le souhaiterait le locuteur. 


\subsection{Malentendu}

Imaginons maintenant le dialogue suivant :

(3) X : Incroyable! Neymar suspendu trois matchs après son altercation avec un supporter.

Y : Tu veux dire que la Commission a été clémente?

$\mathrm{X}$ : Non, je la trouve sévère.

Comme dans le cas précédent, le locuteur $\mathrm{X}$ a l'intention de produire son énoncé Neymar suspendu trois matchs après son altercation avec un supporter de telle manière qu'il évoque l'enchaînement Neymar a eu une altercation avec un supporter pourtant il est suspendu trois matchs, concrétisant le schéma NEG FAUTE PT SANCTION, signifié par le terme constitutif implicite sévère. L'interlocuteur $\mathrm{Y}$ ne reconnaît cette intention que partiellement : il comprend l'énoncé comme évoquant le même enchaînement, mais il se méprend sur le schéma que concrétise cet enchaînement. Selon lui, l'enchaînement concrétise le schéma FAUTE PT NEG SANCTION, signifié par le terme constitutif implicite clément.

On a affaire ici à un simple cas de malentendu qui résulte du fait que l'interlocuteur échoue dans la détermination du schéma que le locuteur considère comme concrétisé par l'enchaînement évoqué.

\subsection{Polémique (1)}

Soit le dialogue suivant :

(4) X : Incroyable ! Neymar suspendu trois matchs après son altercation avec un supporter.

Y : Tu veux dire que la Commission a été sévère?

$\mathrm{X}$ : Oui.

$\mathrm{Y}:$ Moi, je la trouve clémente.

Le locuteur X a l'intention de produire son énoncé Neymar suspendu trois matchs après son altercation avec un supporter comme évoquant l'enchaînement Neymar a eu une altercation avec un supporter pourtant il est suspendu trois matchs et d'attacher cet enchaînement au schéma NEG FAUTE PT SANCTION, signifié par le terme constitutif implicite sévère. L'interlocuteur $\mathrm{Y}$ reconnaît cette intention et comprend l'énoncé comme évoquant le même enchaînement qui concrétise le même schéma. En ce sens, il s'agit ici aussi d'une bonne interprétation. Ce qui distingue les deux cas, c'est que l'interlocuteur $\mathrm{Y}$ attribue ici de surcroît une autre interprétation, la sienne, à l'énoncé de $\mathrm{X}$ : il comprend de son propre point de vue l'énoncé comme évoquant le même enchaînement que $X$, mais il considère cet enchaînement comme concrétisant le schéma FAUTE PT NEG SANCTION, signifié par le terme constitutif implicite clément. Pour les deux interlocuteurs l'énoncé Neymar suspendu trois matchs après son altercation avec un supporter évoque le même enchaînement Neymar a eu une altercation avec un supporter pourtant il est suspendu trois matchs, mais ils attachent cet enchaînement à deux schémas différents: X le présente comme concrétisant le schéma NEG FAUTE PT SANCTION (d'où sévère) et $\mathrm{Y}$ le comprend comme concrétisant le schéma FAUTE PT NEG SANCTION (d'où clément).

Il s'agit ici d'une certaine forme de polémique qui porte sur le schéma que concrétise l'enchaînement évoqué : les deux interlocuteurs, tout en étant du même avis sur 
l'enchaînement évoqué, sont en désaccord sur le schéma concrétisé par l'enchaînement.

\subsection{Polémique (2)} énoncé : libre, parce que le terme constitutif est absent et on peut dès lors attacher l'enchaînement évoqué à un schéma puis un mot de son choix; contraint, dans la mesure où le locuteur peut avoir l'intention plus ou moins manifeste d'attacher l'enchaînement évoqué à un schéma et à un mot précis, et l'interprétation argumentative consistera alors à restituer ce schéma. Argumentative du Lexique ? Non, parce qu'elle ne concerne pas la question sémantique de son organisation - on peut maintenir les hypothèses là-dessus -, mais une autre d'ordre plutôt pragmatique, celle de son utilisation, qui se fait de diverses manières en fonction de l'intention du locuteur. 


\section{BIBLIOGRAPHY}

Anscombre, J.-Cl. et Ducrot, O. (1983) L'Argumentation dans la langue, Bruxelles : Mardaga.

Carel, M. (2011) L'Entrelacement argumentatif, Paris : Honoré Champion.

Carel, M. (2012) «Introduction », in M. Carel (éd.), Argumentation et polyphonie. De Saint-Augustin à Robbe-Grillet, Paris : L'Harmattan, pp. 7-58.

Carel, M. (2015) « Tu seras un homme, mon fils. Un prolongement de la doxa : le paradoxe », in A.-M. Cozma, A. Bellachhab et M. Pescheux (dir.), Du sens à la signification. De la signification aux sens. Mélanges offerts à Olga Galatanu, Bruxelles : Peter Lang, pp. 389-405.

Carel, M. (2017) « Signification et argumentation », Signo, UNISC, vol. 42, n 73, pp. 2-20.

Carel, M. (2019) « Décodage et interprétation argumentatifs », Signo, UNISC, vo. 44, n 80, pp. 2-14.

Carel, M. et Ducrot, O. (1999) « Le problème du paradoxe dans une sémantique argumentative », Langue française, $\mathrm{n}^{\circ} 123$, pp. 6-26.

Christopulos, G. (2018) « Au-delà de l'isotopie », SHS Web of Conferences, vol. 46, article n 06004, $6^{e}$ Congrès Mondial de Linguistique Française. https://doi.org/10.1051/shsconf/20184606004.

Ducrot, O. (1995) « Les modificateurs déréalisants », Journal of Pragmatics, 24, pp. 145-165.

Ducrot, O. (2016) « Présentation de la théorie des blocs sémantiques », Verbum, tome XXXVIII, $\mathrm{n}^{\circ} 1-2$, pp. 53-66.

Frenay, A. et Carel, M. (2019) « Périodes argumentatives et complexes discursifs », Studii de Lingvistică, vol. 9, $\mathrm{n}^{\circ} 1$, pp. 133-150.

Kida, K. (2015) « Prédicat argumentatif et concept ad hoc », Travaux de linguistique, $\mathrm{n}^{\circ} 70$, pp. 121-137.

Kida, K. (2016) « L'anaphore conceptuelle au prisme de la " théorie des blocs sémantiques " ", Discours, 19. https://journals.openedition.org/discours/9217.

Kida, K. (2017) « L'argumentativité de la métaphore dans une sémantique argumentative », in M. Bonhomme, A.-M. Paillet et Ph. Wahl (éd.), Métaphore et argumentation, Louvain-la-Neuve : Éditions Academia, pp. 117-134.

Kida, K. (à paraître) « Le concept de paradoxe », in J. C. Machado (éd.), Cours de sémantique argumentative : des concepts clés, UEMG.

Nishiwaki, S. (2016) Ironie et argumentation : l'exemple de la campagne présidentielle de 2012, thèse de doctorat, EHESS, Paris.

\section{NOTES}

1. On ne distinguera pas l'«aspect argumentatif» et le «quasi-bloc» au sens technique des termes.

2. Pour être plus précis, on devrait dire: schéma doxal. On se reportera avec profit, pour la définition linguistique de la doxa et du paradoxe dans le cadre de la Théorie des Blocs Sémantiques, à Carel (2015, 2017), Carel et Ducrot (1999), Kida (à paraître). 
3. Sauf les cas où plusieurs énoncés développent le schéma d'un même terme à l'intérieur d'une même " période argumentative ». Voir à ce sujet Christopulos (2018), Frenay et Carel (2019).

4. https://www.lemonde.fr/football/article/2019/05/10/football-neymar-suspendu-troismatchs-apres-son-altercation-avec-un-supporteur_5460446_1616938.html (consulté le 18 août 2019).

5. On pourrait songer qu'une quatrième interprétation est possible, selon laquelle l'enchaînement Neymar a eu une altercation avec un supporter donc il est suspendu trois matchs évoqué par l'énoncé concrétise le schéma FAUTE DC SANCTION préfiguré par le terme constitutif implicite faute et c'est ce schéma que le locuteur choisit. Il n'en est rien. L'énoncé reçoit encore une autre interprétation, non moins possible, selon laquelle il l'évoque l'enchaînement Neymar a eu une altercation avec un supporter pourtant il est suspendu trois matchs qui concrétise le schéma FAUTE PT NEG SANCTION préfiguré implicitement par faute, au même titre que le précédent. Entre les deux schémas également préfigurés par le même mot, il n'y a aucune raison de privilégier l'un plutôt que l'autre.

6. Cette conclusion, si elle est bonne, pose un gros problème à la Théorie des Blocs Sémantiques dans la mesure où elle remet en cause son hypothèse fondamentale selon laquelle tout énoncé est paraphrasable par des enchaînements argumentatifs attachés aux schémas qu'ils concrétisent.

\section{ABSTRACTS}

This article aims to develop the notion of "argumentative interpretation" introduced by Carel (2019) in the Theory of Semantic Blocks with two other notions, those of "argumentative decoding" and of "Argumentative Lexical Map". It will be claimed that the argumentative interpretation is subject to constraints whereas Carel seems to give more weight to the freedom that one has to interpret argumentatively an utterance.

Le présent article vise à développer la notion d'« interprétation argumentative » introduite par Carel (2019) dans la Théorie des Blocs Sémantiques avec deux autres notions, celles de « décodage argumentatif» et de "Carte Argumentative du Lexique». Il s'agira de montrer que l'interprétation argumentative est soumise à des contraintes alors que Carel semble donner plus de poids à la liberté qu'on a d'interpréter argumentativement un énoncé.

\section{INDEX}

Keywords: Theory of Semantic Blocks, argumentative semantics, argumentative decoding, Argumentative Lexical Map

Mots-clés: Théorie des Blocs Sémantiques, sémantique argumentative, interprétation argumentative, décodage argumentatif, Carte Argumentative du Lexique 
AUTHOR

KOHEI KIDA

Université Keio

kida@flet.keio.ac.jp 